\title{
EVOLUTIONARY DESIGN: THE APPLICATION OF BIOLOGICAL STRATEGIES IN THE PRODUCT DESIGN PROCESS
}

\author{
NARIMAN G. LOTFI \\ German University in Cairo.
}

\begin{abstract}
The research aimed at understanding how strategies developed by nature endure and sustain organisms and the environment in which they are placed. Accordingly, this understanding of biological strategies led to the development of a product design process that enhances both the innovation and sustainability of the product and its surroundings. An in-depth comparison was discussed of two main strategic directions that shape existing nature-inspired design process; outcome-focused strategies and process-focused strategies. Two processes were developed that follow nature's method of adapting to survive in changing environments which were tested in the product design field. The approach of experimentation was divided into two main parts; case studies and interactive participant labs to experiment and apply the research outcome. Participants from different disciplines and professional backgrounds were involved in both experimentation methods. The methods were analysed using observation, interviews, questionnaires, and focus group discussions. The experiments showed that participants from the design, engineering, and management fields produced sustainable outcomes by applying the proposed processes in their projects. By introducing nature-inspired processes during the start of project development, participants were able to create sustainable solutions. The proposed framework can be applied in the education sectors for design students. It can also be applicable for professional design projects to achieve advanced sustainable outcomes.

Keywords: evolution, product design, processes, sustainability, strategies.
\end{abstract}

\section{INTRODUCTION}

The relationship between design and nature can enhance product design innovation and sustainability by considering processes and survival strategies in nature such as evolution and growth. Existing processes following biological principles include Biomimicry, Bionics, and Evolutionary design, and provide designers and students structures to achieve sustainable designs. However, the intersection of frameworks into the design process is to be addressed. By combining design processes and strategies found in biology a framework can be introduced to aid the product design dynamic.

The aim of this research is to incorporate a nature-inspired strategy into the product design process to develop a framework. This can be introduced to product design students seeking to enhance innovation through nature-inspired design.

\section{LITERATURE REVIEW}

\subsection{Nature-inspired strategies and processes}

Strategies develop for survival and success. McKeown describes a strategy as shaping the future for humans to get to "desirable end with available mean" [1]. Advanced strategies in 
biology are methods of survival common in nature, such as evolution or survival of the fittest. By adapting to environments, strategies allow organisms to coexist and survive sustainably. Existing strategies inspired by nature can be divided into two main categories; outcomefocused strategies and process-focused strategies.

\subsubsection{Outcome-focused strategies}

An outcome-focused strategy follows rules that achieve outcomes inspired by nature. Processes, such as Biomimicry and Bionics, focus on outcomes inspired by nature with elements of nature incorporated to achieve products, systems, services, or buildings.

\subsubsection{Biomimcry}

Developed in 1990 by Janine Benyus, it incorporates nature's knowledge into its framework to ultimately create "conditions conducive to life." Life's principles are categorized into evolve to survive, be resource efficient, adapt to changing conditions, integrate development with growth, be locally attuned and responsive, and use life-friendly chemistry [2].

Figure 1 shows two directions for planning a project or outcome using Biomimicry; biology to design or challenge to biology [3]. The directions depict whether one starts with nature's knowledge or the design challenge first. Ask Nature is a website database with summaries and descriptions of natural strategies to aid professionals to apply nature practically. It collaborates with biologists and people searching for answers from nature. The 'taxonomy' section gathers biologists' knowledge about nature and divides it into groups, allowing users access to information easily and conveniently. Once an inspiration is found, it can be used for an application. Each category provides information about organisms, focusing on a unique aspect about each one mentioning how to apply this information [4].

\subsubsection{Bionics}

The term was introduced by Jack E. Steele in 1958, describing it as "transferring technologies into life-forms", allowing a relationship between artificial and natural in industrial design [5]. According to Werner Nachtigall, Bionics involves "learning from nature as an inspiration for independent technical design" [6]. Specific to design, Bionics is defined as "the structure and the function of living organisms with the aim of acquiring inspiration to design" [7]. It can help develop ideas and meaningful concepts in products. The main purpose of Bionics is to "carry out a benchmark of nature, what is created, tested and evolved over millions of years" [8].
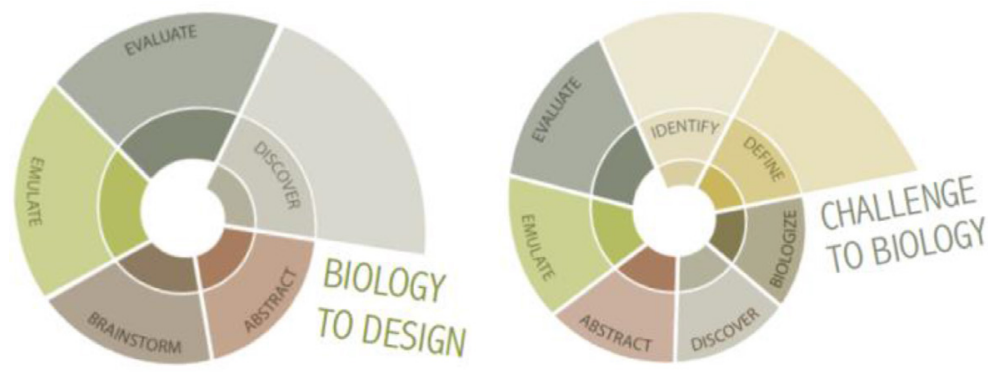

Figure 1: Biomimicry approaches. 


\subsubsection{Process-focused strategies}

In nature, strategies develop for an organism to survive and can be evolution, development or survival of the fittest. These processes take time until they produce a suitable, sustainable outcome capable of surviving in particular intended environments. These strategies include Evolutionary design and can be imitated to create survival processes in designs similar to nature.

\subsubsection{Evolutionary design}

The concept of design evolution relates to scientific theories concerning evolution, and is thought of as "the evolution of ideas" [9] by understanding "principles that lead to a problem solution during evolution and to learn from these principles" [10]. In 2002, Ozcan developed the question: "whether [Darwin] would come up with similar principles of evolution like the selection of the fittest of progression from simplicity to complexity for designed objects." This is believed to be a filter and not as a force, connecting it to the selection filtration process found in design.

Another comparison between objects and evolutionary concepts is nature's need to strive for survival, similar to designs fighting for survival as they compete for resources. According to Langrish, competitions between ideas occur in designers' heads, organizations, and the selective world, suggesting that ideas are naturally selected.

\subsection{Strategic directions in nature-inspired design processes}

These are "two opposing directions" that are available for applying nature to the design process [8]. As previously mentioned, Biomimicry offers two frameworks; the design to nature approach or the nature to design approach. Both offer guidelines and are compared to life's principles. Based on the Aalborg bio-inspired design method developed by Colombo in 2007, the process starts with analyzing a natural system based on its form, structure, and functional principles, and transforming the principle into technical and mechanical terms, to abstract and simplify, making it applicable to design. Finally, new products are established and evaluated, taking into consideration "environmental and economic factors" [11].

\section{METHODOLOGY}

\subsection{Case studies}

Two case studies were developed where different groups of researchers, designers, or students were chosen to address the topic holistically.

\subsubsection{Case study 1: testing strategic directions in design education}

Concepts of the two strategic directions were regarded as metaphors for design approaches introduced to third-year design students at the German University in Cairo. Students were asked to choose one approach to developing mechanisms. Successful approaches abstracted different movements using basic materials to create working mechanisms.

Students used trial-and-error with research and knowledge, developing an Evolutionary process. One successful student explained that he gained an idea first, then abstracted it to consider its functionality, simplistically.

Through observation, as shown in Fig. 2, students were divided into two groups: those who viewed the challenge in an abstract metaphorical sense of how an organism functions in a 

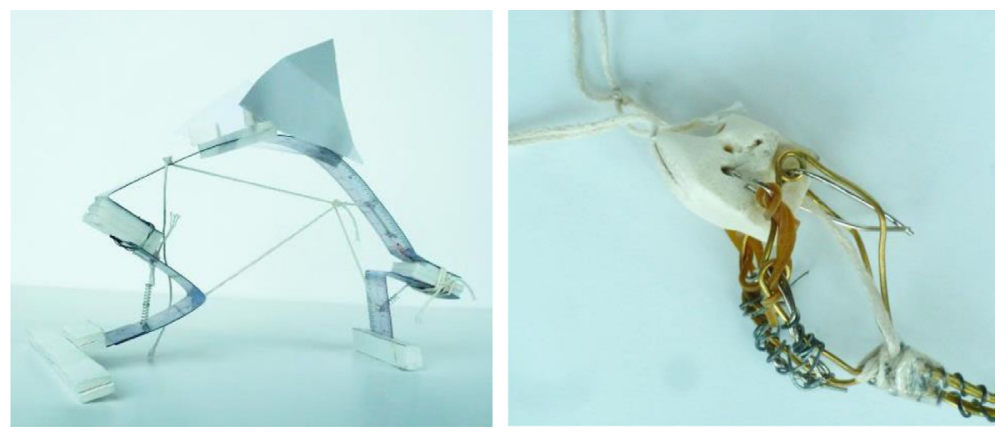

Figure 2: Cat inspired mechanisms. Left, a cat's back's function and right, a cat's claw.

certain way, i.e. jumping of a grasshopper, and cat's back (left), and those who thought of technical aspects in organisms (muscles, tendons, ligaments, bones) whose outcomes functioned similar to the organisms', i.e. cat claw, human elbow (right).

\subsubsection{Case study 2: testing biomimicry in design innovation and research}

The Biomimicry Student Design Challenge is an annual competition set up to encourage participation in the Biomimicry process. In 2012, the challenge involved solving a local or global water issue using nature's knowledge [12]. This case study tested one of the Nature-Inspired Design Strategies first-hand.

By applying nature into the process concepts and ideas were developed faster from a unique perspective. However, a gap was apparent where transitions from nature to design occurred. The team discussed ideas online with biologists which aided in the design development. However, questions needed narrowing down; if nature is observed too deeply a designer loses focus of the goal of the design. Meetings required abstracting and emulating nature to create a design, but who does the abstracting? Images and information provided by Ask Nature were inspiring but knowledge implementation was difficult; without biological knowledge on organisms' functions how can we abstract it?

The design, as shown in Figure 3, was successful in the challenge and won the Grand Prize award in 2013. However, if a designer gets engulfed in understanding organism components used as inspiration it causes designers to lose sight of end users and their needs.

\subsubsection{Conclusion of case studies}

The case studies showed the flexibility of using and applying nature into different approaches such as mechanical development, system development, and strategy development. Nature can be used technically to find core functions of organisms and apply them simplistically. This knowledge allows designers to develop products using reduced material usage, making them practical, affordable, and sustainable.

\subsection{Developing nature-inspired strategies for the design process}

Using the information provided by the case studies two strategies were developed to aid designers implement nature into their approaches. 


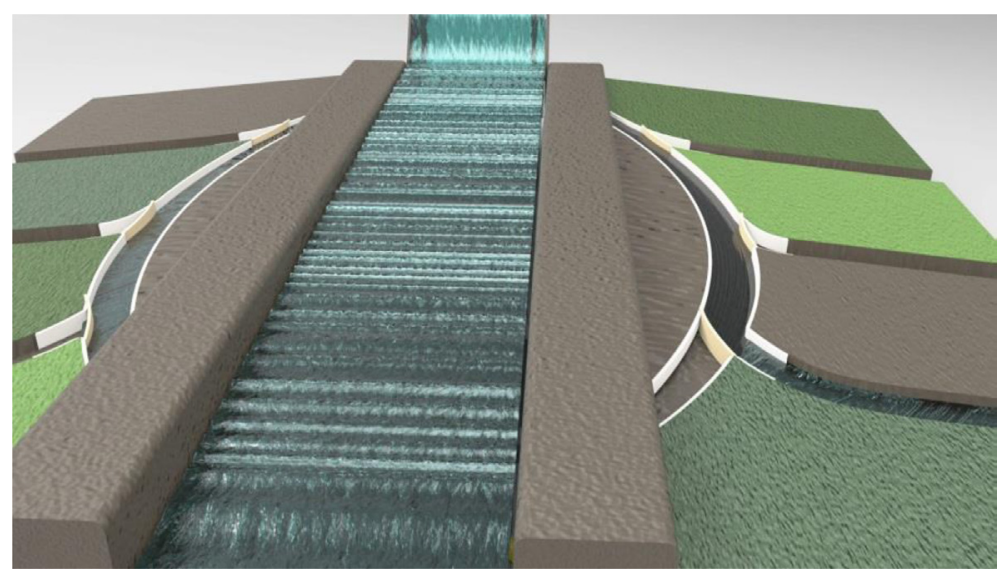

Figure 3: Biomimicry Student Design Challenge outcome in 2012-2013. The design was inspired by the camel and the giraffe to create an efficient water management solution, winning the Grand Prize in 2013 [13].

\subsubsection{Life's principles mapped out in the design process}

This strategy implements life's principles from the Biomimicry methodology into the design process. As illustrated in Figure 4, the standard design process is followed through initially and each stage uses life's principles, allowing designers to solve problems. Each principle was chosen and placed in a certain stage in the process where it would be needed. The design is developed according to the following steps:

1. What is the problem that needs to be solved? This is evaluated against 'using feedback loops' and 'reshuffling information.'

2. The design development takes place and is evaluated against 'how nature uses multifunction' and 'fits form to function.' It is also evaluated against 'using diversity' and 'replicating strategies that work.'

3. Further development occurs and is analysed against 'embodying resistance and maintaining integrity', as well as 'self-organization.

4. Prototyping involves 'being locally attuned' and 'using low energy'.

5. Product launching involves using 'cyclic processes and feedback loops.'

6. The outcome is "fed" through the process once more and is evaluated against each principle again to reduce what is unnecessary.

The outcome of this strategy is a fully analysed, conscious and evaluated design that uses the teachings of "less is more" to create a sustainable and reduced design.

\subsubsection{Testing}

Three participants from different disciplines; product design, management, and engineering, were introduced to the framework and were asked to choose an everyday problem and develop a solution using the framework. The experiment focused on participants' interaction with the strategy.

The outcome showed that using nature as inspiration new ideas are generated for problem-solving. By thinking in an ecosystem sense the group developed a concept in a 


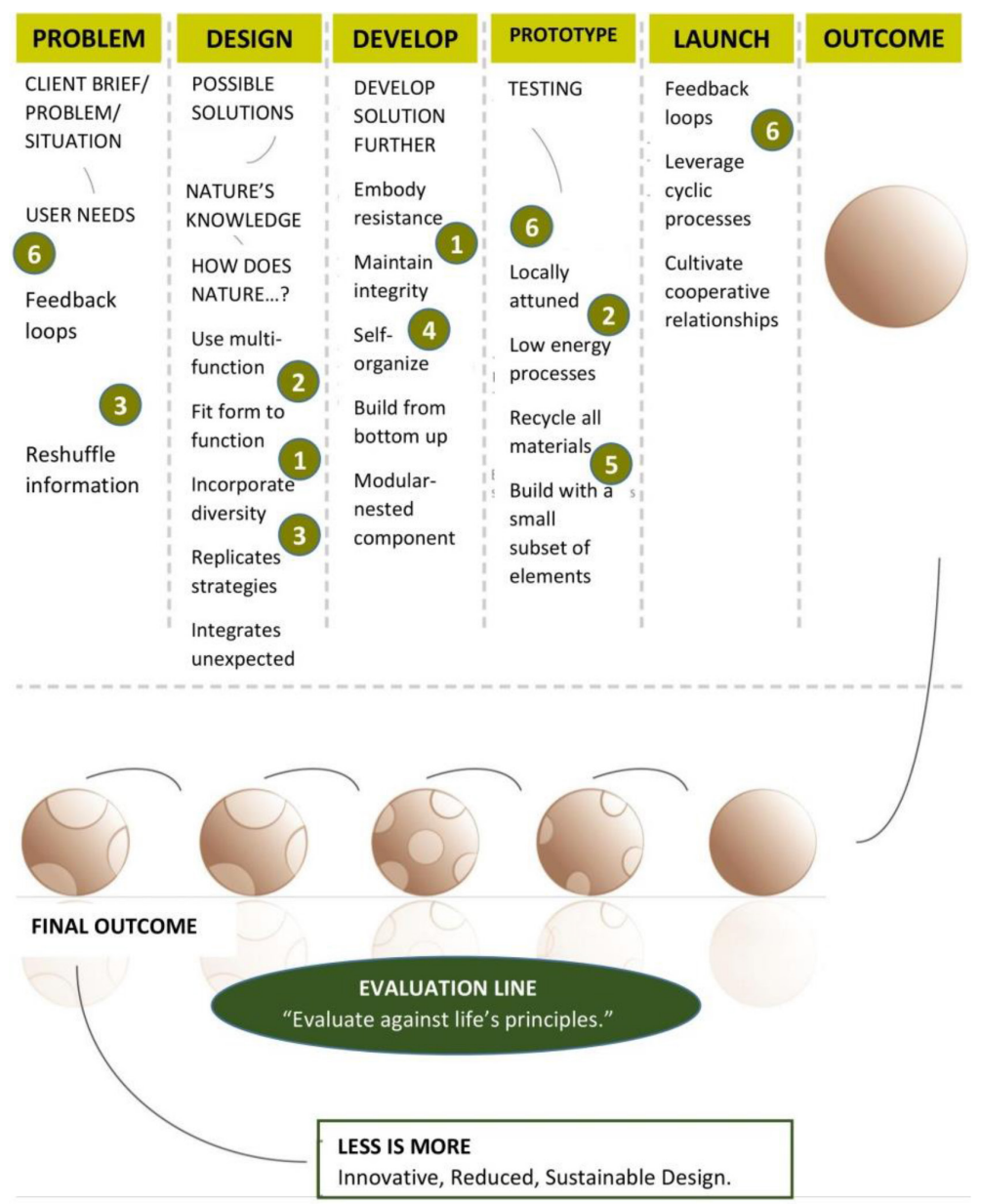

Figure 4: Life's principles process.

sustainable direction. The idea of recycling energy was triggered by the way plants recycle energy to benefit them and their environments. Participants followed a process based on their backgrounds in an interdisciplinary group, yet tasks were not divided. Working together enabled them to generate more ideas and the standard problem-solving process was followed through in a short-term observation.

\subsubsection{Development and discussion}

Challenging participants from different disciplines in a short amount of time proved successful. Supplying them with a set of problems and inspirations restricted thoughts and participants chose their own problem and inspiration. In developing a framework, it should not limit participants but rather give them a set of open tools to develop and evolve their own ideas. The process can be developed using these solutions:

1. Images showing examples of solutions in nature.

2. Explanations about the principles. 
For future testing, this strategy can be tested first with the images then with brief explanations. The test can be analyzed using these criteria:

1. Ease of principle understanding.

2. Clarity of process.

3. Does the process improve the design process?

4. Is the outcome easy to achieve?

5. Is the outcome innovative?

6. Does it become "sustainable"?

7. Is it easy to evaluate against life's principles?

This strategy suggests that the design process is linear and is followed step-by-step. This is not the case and further development needs to be done to fully depict how the design process truly looks like. Although this strategy focuses on Biomimicry research it is not nature-inspired. It does consider life's principles derived from nature, but the process itself is not nature-inspired.

\subsubsection{Evolutionary strategy in the product design process}

By understanding how nature uses survival tactics in its environment an application in design can be developed allowing similar survival of products without disrupting surrounding environments.

This framework, illustrated in Figure 5, focuses on incorporating layers as the concept of nature's survival attitude. The different layers represent the following:

1. The bottom layer contains species of an organism.

2. Through environmental filtration species are reduced in each layer.

3. The top layer contains the fittest species that have adapted to survive.

Accordingly a design platform that symbolizes each layer of products can be incorporated. Impacts from surrounding environments affect each product and it either fails or adapts to survive. Each of the product "species" contains factors that develop it: problem understanding, research, natural inspiration, materials, solution etc. As a product moves along each layer these factors adapt to the effects from the surrounding environment i.e. local materials, client needs, user demands, etc. The contributing external factors; prey, predators, users, climate, and shelter were analyzed as well as the feedback loops between each of the products and the contributors. Each surviving product was observed in its creation through materials, function, concept, and shape. These elements are combined together to adapt the product to survive in the environment it is placed.

The strategy compared the evolutionary process of the survival of the fittest in nature to that of the product design world and developed a 'Natural Philosophy'. It observed contributing factors in the natural world and used them to inspire a filtration system between competing products.

\subsubsection{Development and discussion}

In this illustrated strategy an environment disruptor was not mentioned. In nature, through genetic mutations, we can find organisms that are superior dominating all other organisms, similar to marketplace disruptors and trendsetters. For future development, one can focus on 


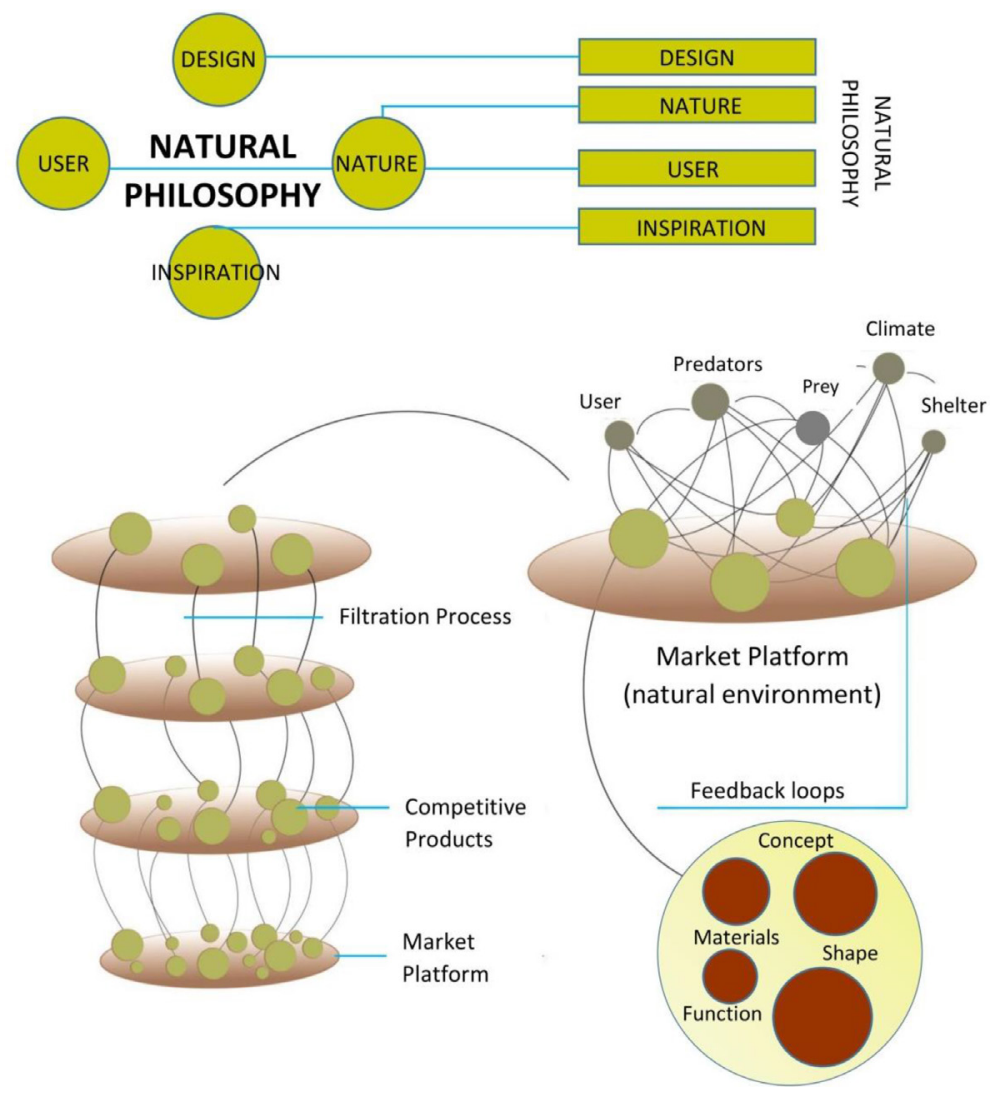

Figure 5: Evolutionary process.

how to create a successful trendsetter based on knowledge of genetic mutation and adaptation. This strategy illustrated similarities between evolution and adaptation in the natural world and that of the artificial world. However, the natural environment is left as it is and the contributors are discussed regarding the natural world. Therefore, for future development, each contributor's effect on the artificial environment needs to be mentioned.

This Evolutionary representation focuses on a long-term approach to design development and depends on both adaptations and communication feedback between product factors. However, if this process is further developed it can capture short-term needs of a product development (communication feedbacks inside a product), as well as the overall evolutionary adaptation process products follow once they are on the market and dealing with their environment.

\section{DISCUSSION}

The Evolutionary design strategy aims at users consciously implementing nature's knowledge and experience into the design process. A flexible yet restrained framework needs to be developed to allow a three-dimensional perspective to outcomes, instead of a linear step-bystep process. A short-term process should be developed that could provide the frame for sustainable achievements. To further develop this strategy the following can be considered: 
- Developing a design package allowing users to learn from nature and solve design needs.

- Communicating benefits of using nature as a consultant to convince users of the strategy and using it through marketing outcome benefits.

- Create dynamic strategies that take into account changes in surroundings and design environment.

- Database providing abstracted organisms could be used as design processes for practical outcomes, developed in interactive software; once an organism's method is entered its strategy of surviving can be abstracted automatically.

\section{CONCLUSION}

The research demonstrated a link between design and nature that can produce design processes inspired by natural systems. These processes create sustainable solutions following processes nature uses. An understanding about interdisciplinary inclusion for solutions to problems has been demonstrated.

The main findings of the research are the achievements of short-term sustainable solutions using a common inspiration participants can use to solve problems, helpful for interdisciplinary work; nature.

This research benefits designers to achieve sustainable, innovative solutions. Design students will be able to understand the design processing and nature-inspired design innovation. Clients of designers can understand the differences between previous designs and sustainable designs achieved using this methodology, and how it can benefit them.

\section{REFERENCES}

[1] McKeown, M., The strategy book. pearson education, Limited, 2012. MercedesClass. net. (2005, June 7), 2012, Bionic Car. Retrieved February 16, 2014, from MercedesClass.net: available at: http://www.mercedesclass.net/design/concept-vehicles/bioniccar/.

[2] Baumeister, D., Introduction to Biomimicry. Retrieved October 15, 2012, from Innovation Conference, 2012, available at: https://www.biomimicrydesignchallenge.com/p/ intro-to-biomimicry.

[3] Biomimicry Institute 3.8. (n.d.). Life's Principles. Retrieved February 27, 2014, from Biomimicry Education Network (BEN): available at: http://ben.biomimicry.net/curricula-and-resources/university- curricula/biomimicry-resource-tools/.

[4] Biomimicry 3.8. (n.d.). Ask Nature. Retrieved February 17, 2014, from Dye Solar Cell Technology: available at: http://www.asknature.org/product/b57e64dd3a2a1a9d36a92a5a51ef7293.

[5] Chiu, W.T. \& Chiou, S.C., Discussion on theories of bionic design. International Association of Design Research, pp. 3635-3643, 2009.

[6] Wahl, D.C., Bionics vs. biomimicry: from control of nature to sustainable participation in nature. Design and Nature: Comparing Design in Nature with Science and Engineering, III, 87, pp. 289-298, 2006.

http://dx.doi.org/10.2495/dn060281

[7] Trotto, A. \& Cianfanelli, E., Beyond bionics: a tool of innovation, optimization and ecology. In Design and Semantics of Form and Movement, eds L. Feijs, S. Kyffin \& B. Young, Victoria University: Wellington and United Kingdom, pp. 113-116, 2006.

[8] Verson, C.A. \& Coelho, D.A., Biologically inspired design: methods and validation. In New Frontiers, eds D.A. Coelho \& D.A. Coelho, InTech: Protugal, pp. 101-120, 2011. 
[9] Langrish, Z.J., Darwinian design: the mimetic evolution of design ideas. Design Issues, 20(4), pp. 4-19, 2004. http://dx.doi.org/10.1162/0747936042311968

[10] Dillmann, R., Albiez, J., Gaßmann, B., Kerscher, T. \& Zöllner, M., Biologically inspired walking machines: design, control and perception. Philosophical Transactions: Mathematical, Physical and Engineering Sciences, 365(1850), pp. 133-151, 2007.

[11] Coelho, A.D. \& Carlos, V.A., An approach to validation of technological industrial design concepts with a bionic character. Recent Researches in Business Administration, Finance and Product Management, pp. 101-122, 2010.

[12] Biomimicry Institute 3.8. Biomimicry Student Design Challenge 2012-2013, 2012, Retrieved November 18, 2013, from Biomimicry Student Design Challenge 20122013: available at: http://2012.biomimicrydesignchallenge.com/.

[13] Lotfi, N., Mogawer, R. \& Refaat, D., Biomimicry circulation system for natural irrigation. Academic Journal of Science, 05(01), pp. 1-18, 2016, available at: http://www. universitypublications.net/ajs/0501/pdf/A6A87.pdf. 